Communications in Physics, Vol. 30, No. 2 (2020), pp. 99-110

DOI:10.15625/0868-3166/30/2/14682

\title{
HIGH-ORDER HARMONIC GENERATION FROM HYDROGEN MOLECULAR ION IN COHERENT SUPERPOSITION STATE
}

\author{
NGOC-LOAN PHAN ${ }^{\dagger}$ \\ Department of Physics, Ho Chi Minh City University of Education, \\ 280 An Duong Vuong Street, District 5, Ho Chi Minh City, Vietnam \\ $\dagger$ E-mail: loanptn@hcmue.edu.vn
}

Received 10 December 2019

Accepted for publication 7 April 2020

Published 26 May 2020

\begin{abstract}
Atom in a coherent superposition state reveals an advantage in the enhancement conversion efficiency of high-order harmonic generation (HHG), which is meaningful in producing attosecond pulses. In this study, we expand to investigate a more complicated system, $\mathrm{H}_{2}^{+}$molecule in the superposition of the ground and second excited states, exposed to an ultrashort intense laser pulse by numerically solving the time-dependent Schrödinger equation. Firstly, we examine the enhancement of HHG from this system. Then, we study the depletion effect on the cutoff energy of HHG spectra with the coherent superposition state. We found that these effects on the $H H G$ from molecules are similar to those from atoms. Finally, we study the signature of the interesting effect, which is absent for atoms - two-center interference effect in the $\mathrm{HHG}$ from $\mathrm{H}_{2}^{+}$in the coherent superposition state. We recognize that the minimum positions in HHG from molecules in the superposition state, and in the pure ground state are the same. Especially, for weak laser intensity, in the HHG with the superposition state, the minimum due to the interference effect is apparent, while it is invisible in the HHG from pure ground state. As a result, in comparison with the ground-state molecule, the coherent molecule can be used as a more accurate tool to determine the internuclear distance of molecule.
\end{abstract}

Keywords: HHG, depletion, enhancement, excited state, interference, superposition state.

Classification numbers: $42.65 . \mathrm{Ky}$.

(C)2020 Vietnam Academy of Science and Technology 


\section{INTRODUCTION}

High-order harmonic generation (HHG) is a highly nonlinear phenomenon when atoms, molecules, or solids interact with ultrashort intense laser pulses [1-3]. The physical mechanism of HHG can be well understood by the three-step model [1], where electron firstly tunnels from the ground state; then, quasi-classically moves in the laser field; finally, recombines into the ground state and emits HHG. The HHG spectrum is characterized by a typical shape with a broad harmonic plateau, which is ended by a cutoff [1].

To date, HHG is the only source for producing attosecond pulses [4], which have many applications in ultrafast science [5,6]. To improve the efficiency and energy of the attosecond pulse, many efforts have been focused on enhancement the intensity and expanding the cutoff of HHG [7-15]. One of the effective methods is using atoms in a coherent superposition of the ground and excited states [11-15]. In this system, the HHG benefits both from a highly ionization probability from the excited state, and a large dipole transition from the continuum into the ground states. As a consequence, the HHG intensity from a coherent superposition state is much enhanced, while its cutoff energy is the same as that of pure ground state [11-14]. Moreover, in our recent study, we have found that in a considerably strong laser that excited state quickly depletes, the cutoff energy of the HHG with superposition state is lower than that with the pure ground state [15]. However, these studies are mostly restricted to atoms [11-15]. Bao et al. have investigated the $\mathrm{HHG}$ emitted from the $\mathrm{H}_{2}^{+}$molecule in the coherent superposition state in a single-cycle laser pulse. They have indicated the possibility of monitoring electron localization dynamics by the dependence of the HHG yield on the laser carrier-envelope phase [16]. However, the enhancement or the depletion effect on the HHG with the coherent superposition state for a such molecule is still questionable and needs to investigate thoroughly.

Recently, many studies have focused on complex systems like molecules since its HHG spectra comprise rich structural information. An interesting effect is interference minimum appeared in HHG spectra, which enables to extract molecular structure [2,17,18]. It should be noted that this two-center interference is absent in the HHG spectra from atoms in the single state, as well as in the coherent superposition state. This effect has been widely investigated for oneelectron [2,17, 18], multi-electron [19], and vibrate [20] molecules. However, the interference effect in HHG spectra from molecules in a coherent superposition state is undiscovered.

Our goal in this paper is to study the HHG spectra emitted from $\mathrm{H}_{2}^{+}$molecule in a coherent superposition of the ground and second excited states. We examine, in turn, the enhancement, depletion, and interference effects in HHG spectra from this system. For simplicity, the onedimensional (1D) $\mathrm{H}_{2}^{+}$molecule is chosen. The HHG is simulated by numerically solving the time-dependent Schrödinger equation (TDSE). Besides, classical simulation is also performed to explain the obtained results.

The paper is arranged as follows. Section II describes the theoretical background, including the TDSE method, analytical model of atoms in the coherent superposition state, and the interference effect in molecules. Section III presents our results and discussion about $\mathrm{HHG}$ from $\mathrm{H}_{2}^{+}$ molecule. Section IV contains a conclusion. 


\section{THEORETICAL BACKGROUND}

\section{II.1. Numerical method of solving time-dependent Schrödinger equation}

When a linear molecule exposed to a linearly polarized laser, it rapidly aligns along the direction of the electric field. Moreover, the ionized electron moves along the polarization direction. Therefore, we employ the one-dimensional model of $\mathrm{H}_{2}^{+}$molecule for computational- and time-saving.

The HHG is calculated from the wave-function, which is obtained by the numerical method of solving the time-dependent Schrödinger equation. For convenience, we use the atomic units with $\hbar=m_{e}=e=1$, whose mass, charge, and energy are written in units of the electron mass, elementary charge, and Hartree energy [21]. The equation has the following form

$$
i \frac{\partial}{\partial t} \Psi(x, t)=\left(-\frac{\partial^{2}}{2 \partial x^{2}}+V_{C}(x)+V_{i}(x, t)\right) \Psi(x, t),
$$

where $x$ is the electron coordinate respect to the center of mass of the two nuclei. The soft Coulomb potential [22] can be written as

$$
V_{C}(x)=-\frac{1}{\sqrt{\left(x-\frac{R}{2}\right)^{2}+a}}-\frac{1}{\sqrt{\left(x+\frac{R}{2}\right)^{2}+a}}+\frac{1}{R} .
$$

Here, $R=2.0$ a.u. is the internuclear distance; $a=1.55$ is the softening parameter which is chosen to ensure the agreement of its energy with the real value [23]. The energy of the $|1 s\rangle$ and $|3 s\rangle$ states are 1.1 a.u. $(29.4 \mathrm{eV})$ and 0.44 a.u. $(12.0 \mathrm{eV})$. The interaction between the molecule and laser is described in the length gauge as

$$
V_{i}(x, t)=x E(t)
$$

The polarization direction of laser aligns along $x$ axis. The electric field has following form

$$
E(t)=E_{0} \sin ^{2}\left(\frac{\pi t}{\tau}\right) \cos \left(\omega_{0} t+\pi\right)
$$

where $E_{0}, \tau, \omega_{0}$, are the field amplitude, pulse duration, and laser carrier frequency, respectively. In this study, we use lasers with the duration of five optical cycles, wavelength of $1600 \mathrm{~nm}$, and different intensities for all calculations.

The time-dependent wave function is found by applying the second-split operator technique

$$
\Psi(x, t+\Delta t)=\exp \left(-i \frac{V(x, t)}{2} \Delta t\right) \exp (-i \hat{T} \Delta t) \exp \left(-i \frac{V(x, t)}{2}\right) \Psi(x, t)+O\left(\Delta t^{3}\right),
$$

where $V(x, t)=V_{C}(x)+V_{i}(x, t)$ is the total potential; and $\hat{T}$ is the kinetic energy operator [24]. The initial wave function, i.e., at time $t=0$, is obtained by using the imaginary time propagation method [25]. In this study, we assume that initially, the molecule $\mathrm{H}_{2}^{+}$is prepared in the coherent superposition of the ground $|1 s\rangle$ and the second excited $|3 s\rangle$ states with equal weighted contribution

$$
\Psi(x, t=0)=\frac{\Psi_{|1 s\rangle}(x, t=0)+\Psi_{|3 s\rangle}(x, t=0)}{\sqrt{2}} .
$$

In fact, the coherent superposition of the two states can be experimentally archived by the multiphoton excitation or one-photon resonant process [26]. In our calculation, we use a grid of 
3200 a.u. with step of 0.1 a.u. The time step is 0.07 a.u. All these parameters have checked to ensure the convergence.

After obtaining the time-dependent wave function, the ionization probability is derived as follows

$$
P(t)=\int_{S}|\Psi(x, t)|^{2} d x
$$

where the ionization region $S$ is defined as $S:|x|>20$ a.u. The instantaneous ionization rate is the differential of the ionization probability

$$
R_{|n s\rangle}(t)=\frac{d}{d t} P_{|n s\rangle}
$$

The dipole acceleration is

$$
a(t)=-\left\langle\Psi(x, t)\left|\frac{d V(x, t)}{d x}\right| \Psi(x, t)\right\rangle .
$$

Its Fourier transformation gives the HHG signal

$$
S(\omega) \propto\left|\int_{0}^{\tau} a(t) e^{-i \omega t} d t\right|^{2} .
$$

\section{II.2. Analytical model for atom in coherent superposition state}

A simple analytical model based on the strong-field approximation has been proposed to explain the enhancement of the HHG from atom with a coherent superposition state $[11,12,14]$. Here, we briefly recall some main ideas.

For a system with a coherent superposition of the ground (denoted as $g$ ) and excited $(e)$ states in an intense laser, its dipole acceleration can be separated into four distinct terms

$$
a(t)=a_{g g}(t)+a_{e e}(t)+a_{g e}(t)+a_{e g}(t) .
$$

Here, $a_{g g}(t)$ and $a_{e e}(t)$ are the incoherent terms in which electron recombines to the state where it ionizes from. The left two terms are the interference ones. $a_{g e}(t)$ describes the quantum path that electron ionizes from the ground state, then recombines into the excited state. On the contrary, $a_{e g}(t)$ raises the path that electron releases from the excited state but recombines to the ground state. Depending on the laser's parameters, the roles of these components exhibit differently [11$13,15]$.

\section{II.3. Interference effect}

Unlike for the case of atoms whose HHG intensity in the plateau region is almost unchanged, the plateau of the HHG spectra from molecules contains minimums. The minimum is attributed to the destructive interference of the harmonics emitted from the different atomic centers of a molecule $[2,17,18]$. Based on the two-center model [2], the destructive interference of a bonding molecule like $\mathrm{H}_{2}^{+}$occurs when the Bragg condition is satisfied

$$
R \cos \theta=(2 n+1) \lambda / 2,
$$

where $n=0,1, \ldots ; \theta$ is the angle between the molecular axis and the electric polarization vector of a linear laser; and $\lambda=2 \pi /|\mathbf{k}|$ is the wavelength of the returning electron. The value of the wave vector is $|\mathbf{k}|=\sqrt{2 N \omega_{0}}$, where $N$ is the harmonic order. For $1 \mathrm{D}$ calculation, $\theta=0^{\circ}$. From 
the Bragg formula, one can easily predict the harmonics occurring destructive interference for 1D molecule as

$$
N_{i n t}=\frac{(2 n+1)^{2} \pi^{2}}{2 R^{2} \omega_{0}}
$$

\section{RESULTS AND DISCUSSION}

In this section, we present the $\mathrm{HHG}$ spectra from $\mathrm{H}_{2}^{+}$molecule in the coherent superposition of the ground $|1 s\rangle$ and second excited $|3 s\rangle$ states. In our calculation, we assumed that initially, the contributions of the two states are equal since this choice supplies the maximum HHG conversion efficiency $[12,15]$. We in turn discuss the three effects - enhancement, depletion, and interference, in the $\mathrm{HHG}$ spectra from $\mathrm{H}_{2}^{+}$molecule in the coherent superposition state.

\section{III.1. Enhancement of $\mathbf{H H G}$ from $\mathrm{H}_{2}^{+}$molecule in coherent superposition state}

Figure 1 shows the $\mathrm{HHG}$ from $\mathrm{H}_{2}^{+}$in the coherent superposition state $(|1 s\rangle+|3 s\rangle) / \sqrt{2}$ (denoted as $|1 s\rangle+|3 s\rangle$ ) when interacting with the laser with intensities of $1 \mathrm{I}_{0}, 3 \mathrm{I}_{0}$, and $6 \mathrm{I}_{0}$, where $\mathrm{I}_{0}=10^{14} \mathrm{~W} / \mathrm{cm}^{2}$. For comparison, the HHGs from the pure ground state $|1 \mathrm{~s}\rangle$ are also presented. The results indicate that the HHG emitted from the molecule in the coherent superposition state has the same property as that generated from atom. In particular, the HHG of the superposition state is much enhanced compared to that of the pure ground state. Moreover, for weak laser where the excited state does not deplete, or slowly depletes as used in Fig. 1(a), the cutoff energy of the HHG of the superposition state as large as that of the pure ground state. It should be noted that the cutoff position is determined as the end of the plateau region. After the cutoff, the HHG intensity is dramatically dropped [1].

To understand this similarity, we discuss the enhancement mechanism of the atom in the coherent superposition state by employing the analytical model presented in Subsec. II.2. According to Eq. (11), the harmonic generation is caused by four quantum paths. Among them, the interference term $a_{e g}(t)$, where electron releases from the excited state and then recombines into the ground state, is much greater than others and plays a crucial role in the enhancement of HHG. This enhancement is caused by the twofold reason - (i) electron easily ionizes from the excited state because of its small ionization potential $[13,15,27]$, and (ii) probability of recombination into the ground state is higher than into the excited one [12]. Meanwhile, the HHG from the pure ground state is raised by the electron, which is ionized from and is recombined to the ground state, i.e., $a_{g g}(t)$. Therefore, its HHG efficiency is much lower than that of the coherent atom. This explanation also validates in the case of molecules. In short, a molecule in a coherent superposition state also has an advantage in improving the HHG conversion efficiency and ensuring large cutoff energy thanks to the interference dipole acceleration $a_{e g}(t)$.

Figure 1 also shows that with increasing the laser intensity, the enhancement of the HHG from the coherent molecule is reduced. For the laser intensity of $1 \mathrm{I}_{0}, 3 \mathrm{I}_{0}$, and $6 \mathrm{I}_{0}$, the magnification by the superposition state is respectively about 7,3 , and 1 order. This tendency is similar to the case of atom [15], where the reason is the reduction of the survival probability (which compensates with the ionization probability presented in Fig. 3(a)-(c)) of the ground state, leading to the decrease of the component $a_{e g}(t)$. Meanwhile, with increasing the laser intensity, the HHG efficiency of the ground state is rapidly enhanced due to the increase of the ionization probability. These facts narrow the gap between the intensity of the HHG spectra from the molecule with the superposition 


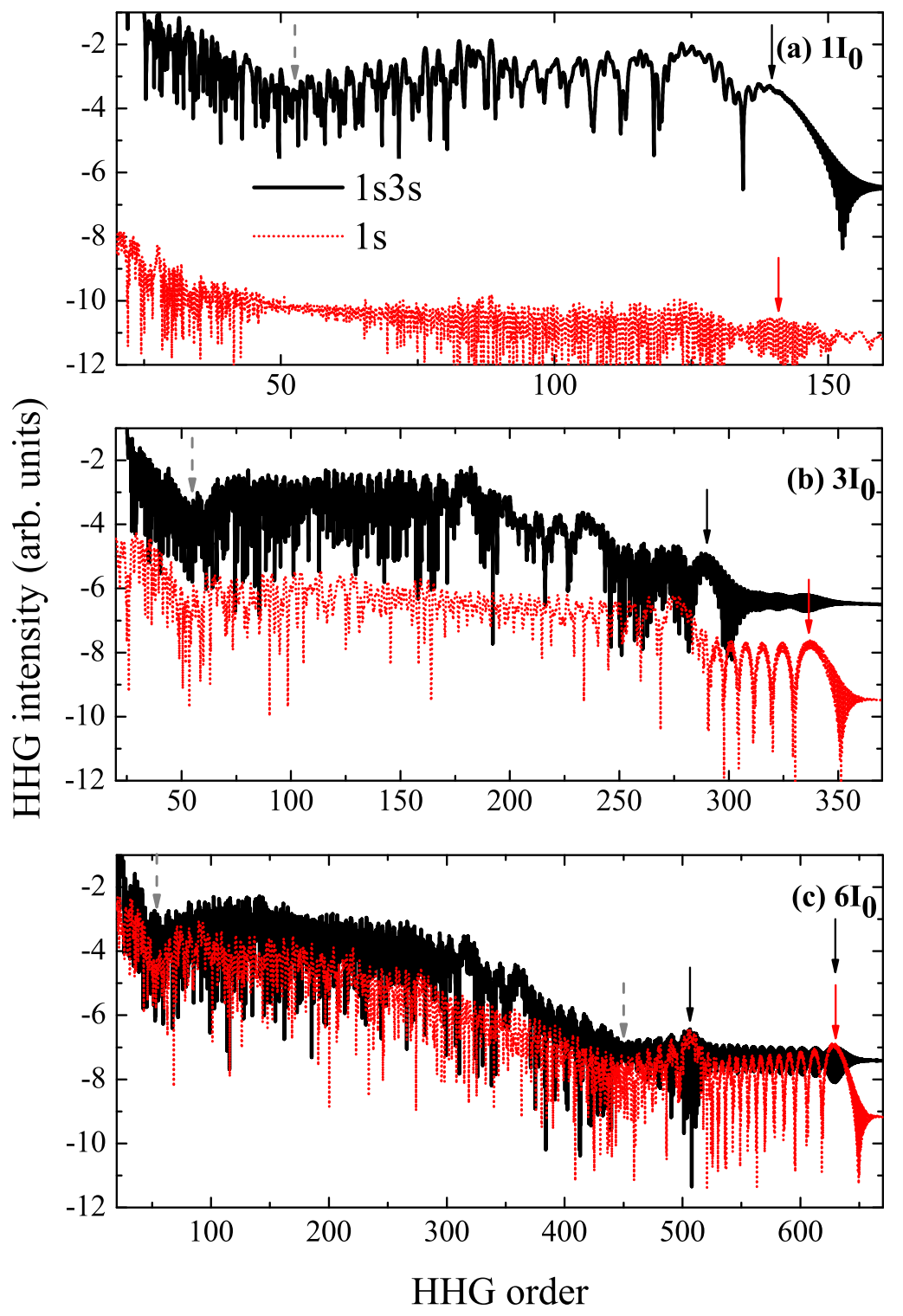

Fig. 1. $\mathrm{HHG}$ spectra from $\mathrm{H}_{2}^{+}$in the coherent superposition state $(|1 s\rangle+|3 s\rangle) / \sqrt{2}$ (black solid curve), and pure ground state $|1 s\rangle$ (red dotted curve). 5-cycle laser with wavelength of $1600 \mathrm{~nm}$, and intensities of $1 \mathrm{I}_{0}(\mathrm{a}), 3 \mathrm{I}_{0}(\mathrm{~b})$, and $6 \mathrm{I}_{0}(\mathrm{c})$, where $\mathrm{I}_{0}=10^{14} \mathrm{~W} / \mathrm{cm}^{2}$. Solid and dash arrows respectively point to the positions of cutoff and destructive interference.

state and the pure ground state. Specifically, for a much strong laser with an intensity of $6 \mathrm{I}_{0}$ (Fig. 1(c)), the harmonics from $506^{\text {th }}$ order of the coherent molecule have the comparable intensity with that of the pure ground state molecule. This phenomenon will be discussed in the next section. 


\section{III.2. Depletion effect in HHG from $\mathrm{H}_{2}^{+}$molecule in coherent superposition state}

Now we investigate the influence of the depletion from the excited state on the cutoff energy of the $\mathrm{HHG}$ with a coherent superposition state for $\mathrm{H}_{2}^{+}$molecule. From Fig. 1, we realize that for $\mathrm{H}_{2}^{+}$molecule, the changing tendency of the cutoff energy in HHG of superposition state with laser intensity is the same as for the case of atom found in our previous study [15]. Specifically, the laser intensity can be divided into three regions, where the correlation between cutoff energies of HHGs of $\mathrm{H}_{2}^{+}$in the superposition state and in the pure ground state reveals differently. For the weak laser with intensity $1 \mathrm{I}_{0}$ as shown in Fig. 1(a), the HHG with $|1 s\rangle+|3 s\rangle$ state has cutoff energy as large as the one of the pure ground state. For the stronger laser with $3 \mathrm{I}_{0}$ in Fig. 1(b), the cutoff energy of the superposition state is much less than that of the pure ground state. However, for the more stronger laser $6 \mathrm{I}_{0}$ as indicated in Fig. 1(c), the HHG with superposition state has the cutoff, again, as same as the one of the ground state. Moreover, the HHG spectrum contains two plateaus with two cutoffs, as shown by black arrows in Fig. 1(c). To easy illustration, we express harmonics at the cutoff in Table 1 .

Table 1. The cutoff by TDSE calculation for 5-cycle laser with wavelength of $1600 \mathrm{~nm}$ and various intensities; and predicted by classical cutoff laws $I_{p}+3.17 U_{p}$ and $I_{p}+$ $2.68 U_{p}$.

\begin{tabular}{cccc}
\hline Intensity & $I_{p}+3.17 U_{p}$ & $I_{p}+2.68 U_{p}$ & TDSE \\
\hline $1 I_{0}$ & $\mathbf{1 4 7}$ & 132 & $\mathbf{1 4 1}$ \\
$3 I_{0}$ & 342 & $\mathbf{2 9 7}$ & $\mathbf{2 9 1}$ \\
$6 I_{0}$ & $\mathbf{6 3 5}$ & 544 & $506, \mathbf{6 3 0}$ \\
\hline
\end{tabular}

To interpret the response of the cutoff energy of HHG in the coherent superposition state of $\mathrm{H}_{2}^{+}$molecule to the laser intensity, we apply the same procedure employing for the atom case [15]. Since the cutoff energy of harmonics is directly related to the kinetic energy of returning electron when it meets the parent ion, we calculate this quantity by the classical simulation $[1,15,28]$ and present in Fig. 2. It is showed that the kinetic energy strongly depends on the ionization time. If electron ionizes at instance $t_{1} \approx 1.53 T_{0}$, or $t_{2} \approx 2.05 T_{0}$, where $T_{0}$ is a optical cycle, then its maximum kinetic energies are respectively $2.68 U_{p}$ and $3.17 U_{p}$, where $U_{p}=E_{0}^{2} / 4 \omega_{0}^{2}$ is the ponderomotive energy of electron in the laser field. The corresponding cutoff energies when electron recombines into the ground state with ionization potential $I_{p}$ are

$$
N_{\text {cutoff }} \omega_{0}=I_{p}+2.68 U_{p}
$$

and

$$
N_{\text {cutoff }} \omega_{0}=I_{p}+3.17 U_{p}
$$

Commonly, the cutoff energy obeys the cutoff law Eq. (15) when the depletion is negligible. However, as shown in the left panels in Fig. 3, the excited state rapidly depletes after about $2.5 T_{0}$, $1.8 T_{0}$ and $1.3 T_{0}$ for laser intensity of $1 \mathrm{I}_{0}, 3 \mathrm{I}_{0}$, and $6 \mathrm{I}_{0}$, respectively. After depletion moment, there is no more electron can ionize, therefore, the cutoff law Eq. (15) is not always satisfied. 


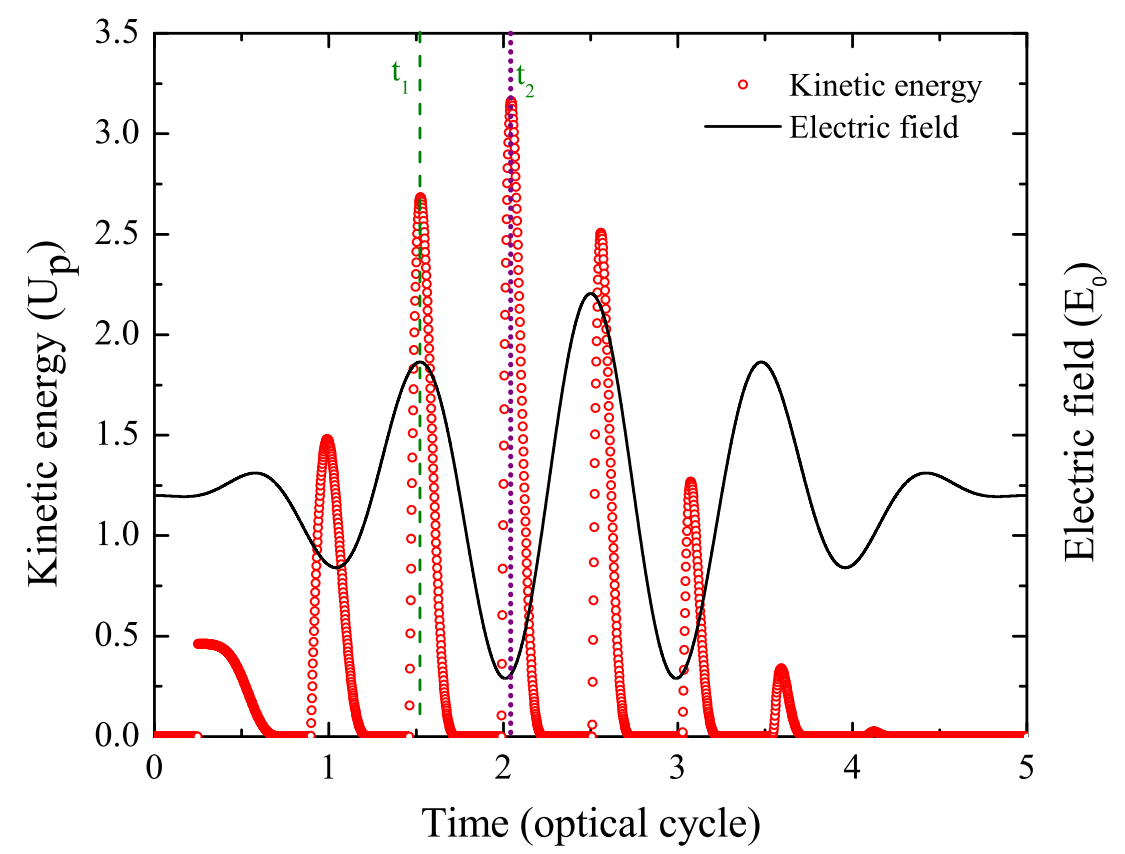

Fig. 2. Kinetic energy of the returning electron (red circles) and the electric field of laser (black curve). Green and violet vertical lines exhibit the two instances $t_{1}$ and $t_{2}$ corresponding to the maximum kinetic energy of returning electron $2.68 U_{p}$ and $3.17 U_{p}$.

For more convenient, we express the ability of ionization at each moment by the ionization rate and indicate in Fig. 3. Now we use the time-dependent ionization rate to interpret the cutoff energy of $\mathrm{HHG}$ from $\mathrm{H}_{2}^{+}$in the coherent superposition state. For the molecule in the coherent superposition state, the explanation of the depletion effect on the cutoff energy is similar to for the atomic case. Indeed, (i) for weak laser with intensity $1 \mathrm{I}_{0}$, the ionization rate from the $|3 s\rangle$ state at instance $t_{2}$, i.e, $R_{|3 s\rangle}\left(t_{2}\right)$ is none zero; therefore, the electron ionizes at this moment recombines into the ground state leads to cutoff law Eq. (15), according to $147^{\text {th }}$ order. This value considerably is consistent with the TDSE result, as shown in Table 1. (ii) However, for stronger laser $3 \mathrm{I}_{0}$, the ionization rate from the excited state is negligible at $t_{2}$ while it is nonzero at $t_{1}$. Therefore, the maximum kinetic energy of the returning electron is $2.67 U_{p}$, leading to the cutoff law Eq. (14). Due to the depletion, this cutoff of the HHG with $|1 s\rangle+|3 s\rangle$ state is much lower than that of the pure ground state satisfying the Eq. (15). (iii) For laser with intensity $6 \mathrm{I}_{0}$, both $R_{|3 s\rangle}\left(t_{2}\right)$ and $R_{|3 s\rangle}\left(t_{1}\right)$ are not higher than zero; thus, the first cutoff is $506^{\text {th }}$ order, much lower than the predicted by Eq. (14) as exhibited in Table. 1. Moreover, $R_{|1 s\rangle}\left(t_{2}\right)$ is considerably high, that ground state, or $a_{g g}(t)$, significantly evolves in HHG and gives the cutoff, $630^{\text {th }}$ order, is consistent with the predicted one by Eq. (15). As a result, $a_{g g}(t)$ causes the second plateau that lasts from $506^{\text {th }}$ to $630^{\text {th }}$ orders. As a consequence, the HHG plateau from $506^{\text {th }}$ order of the coherent molecule has 
the same HHG intensity with that of the molecule in the pure ground state, as shown in Fig. 1(c). In summary, for molecules, the depletion behavior with increasing laser intensity is similar to that of atoms.
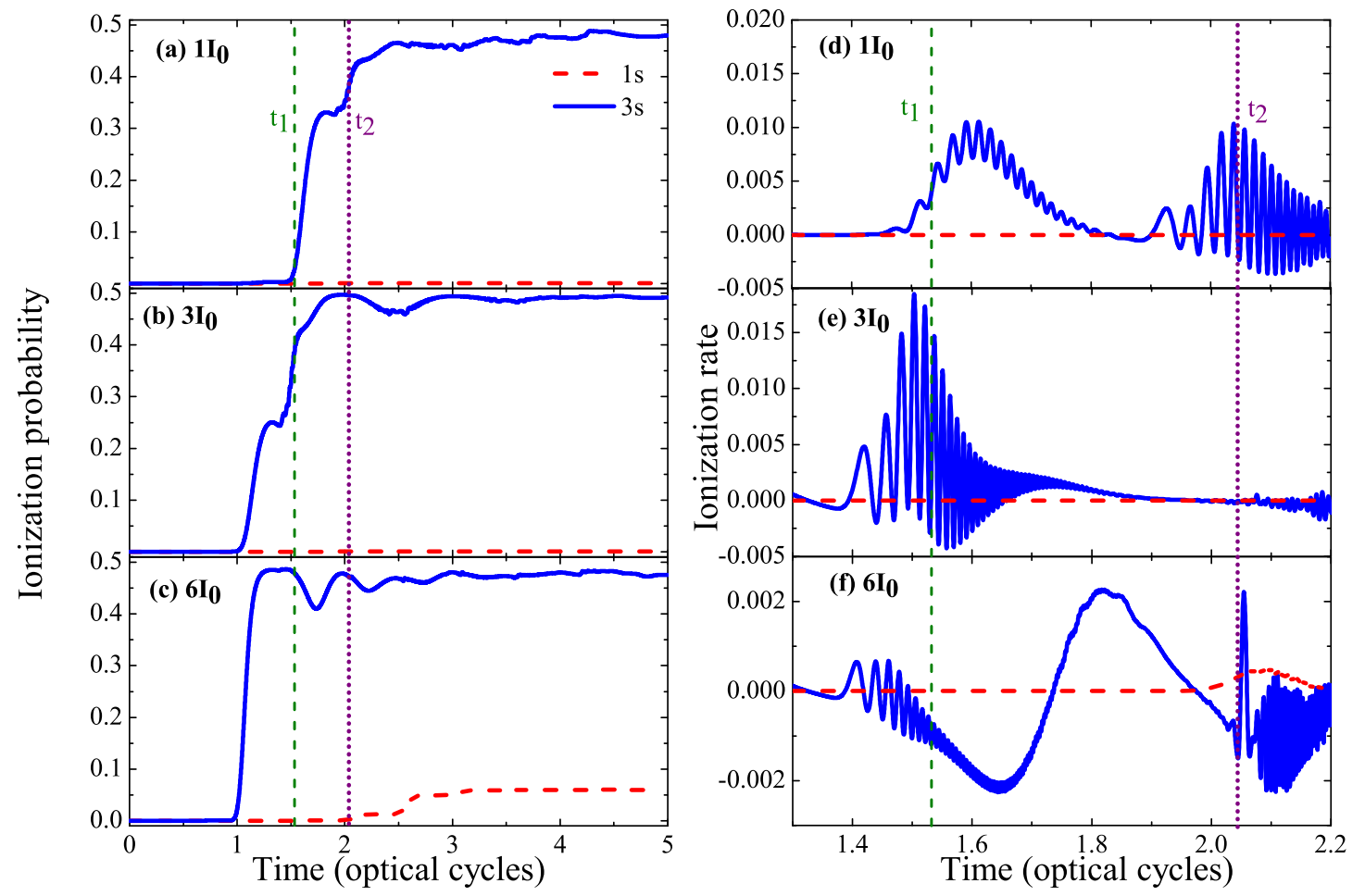

Fig. 3. Ionization probability (left panels) and ionization rate (right panels) from $|1 s\rangle$ (red dashed line) and $|3 s\rangle$ when $\mathrm{H}_{2}^{+}$in the superposition state $(|1 s\rangle+|3 s\rangle) / \sqrt{2}$ of $\mathrm{H}_{2}$ molecule. Laser parameters are the same as used in Fig. 1.

\section{III.3. Interference effect in $\mathrm{HHG}$ from $\mathrm{H}_{2}^{+}$molecule in coherent superposition state}

Unlike HHG from atoms (except Cooper minimum in argon [29]), HHG spectra from molecules contain distinctive harmonics where its intensity is destructive or constructive. This phenomenon is called interference effect well explained by the two-centered model $[2,17]$.

In Fig. 1, we recognize that in the $\mathrm{HHG}$ from $\mathrm{H}_{2}^{+}$molecule in both cases - coherent superposition, and pure ground states, there are apparent minimums whose positions are presented by dash arrows. For the weak laser with intensity of $1 \mathrm{I}_{0}$ as indicated in Fig. 1(a), the HHG of pure ground state does not contain a minimum while it clearly appears in harmonic spectra with superposition state. For stronger laser $3 \mathrm{I}_{0}$, see Fig. 1(b), the minimum's position in HHG with superposition state is the same as that of the pure ground state. A similar scenario occurs for more stronger laser with intensity of $6 \mathrm{I}_{0}$, as shown in Fig. 1(c), not only for the first minimum but also for the second one. The appearance of the second minimum is due to the expanding of the cutoff. Noticeably, the minimum is almost unchanged with the changing of the laser parameters. Besides, 
the minimum positions from the TDSE are considerably consistent with the predicted ones by the Bragg formula Eq. (12) with error less than about $20 \%$. This discrepancy is caused by the fact that the Bragg formula is not good for the "unreal" 1D molecular model [2]. For a clear illustration, we also present these minimum positions in Table 2.

Table 2. The harmonic order at the destructive interference according to predicting Bragg formula, and TDSE calculation for HHG with pure ground, and coherent superposition states. The lasers with parameters as same as in Fig. 1 are used.

\begin{tabular}{c|cc|cc|cc}
\hline \multirow{2}{*}{} & \multicolumn{2}{|c|}{ Bragg law } & \multicolumn{2}{|c|}{ TDSE $|1 s\rangle$} & \multicolumn{2}{c}{ TDSE $|1 s\rangle+|3 s\rangle$} \\
\cline { 2 - 7 } & $\mathrm{n}=0$ & $\mathrm{n}=1$ & $\mathrm{n}=0$ & $\mathrm{n}=1$ & $\mathrm{n}=0$ & $\mathrm{n}=1$ \\
\hline $1 \mathrm{I}_{0}$ & 43 & 385 & - & - & 52 & - \\
$3 \mathrm{I}_{0}$ & 43 & 385 & 55 & - & 55 & - \\
$6 \mathrm{I}_{0}$ & 43 & 385 & 52 & 450 & 52 & 450 \\
\hline
\end{tabular}

To clarify this observation, we recall the main idea of the two-center model $[2,18]$. Accordingly, the returning electron wave-packet, considered as plane waves, recombines into each molecular center and raises harmonic signals. The interference of out-of-phase harmonic signals from molecular centers leads to the appearance of the minimum in HHG spectra. Thus, the interference effect reflects the property of the multicenter molecule upon the recombination; as a consequence, the harmonic energy occurring the interference does not depend on the laser parameter as described by Eq. (12).

The above-mentioned explanation can also be applied for the case of a molecule in the superposition state because of twofold reason. Firstly, the interference effect is mainly attributed to the recombination step. Secondly, the harmonic generation of the superposition state is crucially caused by the recombination to the ground state of the electron ionized from the excited state. Therefore, the recombination step is the same for both HHGs from superposition state, and pure ground state. As a consequence, the destructive interference effect, leading to the minimum position, in their HHG spectra are the same.

However, for weak laser such as used in Fig. 1(a), the minimum can not be observed in the HHG from the ground state, but is noticeable in the one of superposition state. We claim that, as shown in Fig. 3(a), the ionization from the pure ground state is too small that its HHG is considerably weak, leading to the invisibility of interference minimum in the spectra. Meanwhile, the coherent superposition state takes advantage of high ionization probability from the excited state; as a consequence, the HHG is much enhanced, and the minimum position is clearly visible. In short, we conclude that for a weak laser, the superposition state makes the destructive interference effect in HHG clearer compared to the pure ground state.

\section{CONCLUSIONS}

In summary, we have investigated three effects - enhancement, depletion, and interference in the HHG from the $1 \mathrm{D} \mathrm{H}_{2}^{+}$molecule in the coherent superposition of the ground and second 
excited state with an equal weighted contribution by the TDSE method and obtained the following results.

Firstly, as atoms, the $\mathrm{H}_{2}^{+}$molecules with the coherent superposition state also give enhanced HHG spectra with large cutoff energy as same as the pure ground state. This enhancement is attributed to the interference term of induced dipole acceleration $a_{g e}(t)$ where electron ionizes from the excited state and then recombine into the ground state. Therefore, the molecule in the coherent superposition can be a promising tool for producing attosecond pulse with high energy and powerful efficiency.

Secondly, the depletion of the excited state affects the cutoff energy of HHG spectra of $\mathrm{H}_{2}^{+}$ molecule. For molecules, the changing tendency of the cutoff with increasing laser intensities is similar to that for atoms. Indeed, for weak laser, the HHG with superposition state has the same cutoff energy with the pure-ground-state's one. For stronger laser, the cutoff of HHG with the superposition state is lower than that of the pure ground state due to rapid depletion. For more stronger laser, the cutoff of the superposition state, again, fits the one of the pure ground state. Moreover, the HHG with superposition state has two distinct plateaus.

Finally, unlike for the case of the atom, the $\mathrm{HHG}$ spectra from $\mathrm{H}_{2}^{+}$molecule in the coherent superposition state contain minimums due to the destructive interference effect. The position of these minimums are the same with those of HHG from molecule in the pure ground state. Moreover, for weak laser intensity, the coherent superposition state makes the destructive interference effect more apparent than the pure ground state. As a consequence, the internuclear distance determined from the position of the destructive minimum of HHG from a coherent molecule is more accurate compared to the one from the pure ground state.

\section{ACKNOWLEDGMENT}

We thank Prof. Van-Hoang Le for the useful discussion. This research is funded by Vietnam National Foundation for Science and Technology Development (NAFOSTED) under Grant No. 103.03-2018.60.

\section{REFERENCES}

[1] M. Lewenstein, P. Balcou, M. Y. Ivanov, A. L’Huillier and P. B. Corkum, Phys. Rev. A 49 (1994) 2117.

[2] M. Lein, N. Hay, R. Velotta, J. P. Marangos and P. L. Knight, Phys. Rev. A 66 (2002) 023805.

[3] S. Ghimire and D. A. Reis, Nat. Phys. 15 (2019) 10.

[4] J. Li, X. Ren, Y. Yin, K. Zhao, A. Chew, Y. Cheng, E. Cunningham, Y. Wang, S. Hu, Y. Wu, M. Chini and Z. Chang, Nat. Commun. 8 (2017) 186.

[5] E. Goulielmakis, M. Schultze, M. Hofstetter, V. S. Yakovlev, J. Gagnon, M. Uiberacker, A. L. Aquila, E. M. Gullikson, D. T. Attwood, R. Kienberger, F. Krausz and U. Kleineberg, Science 320 (2008) 1614.

[6] P. M. Kraus, B. Mignolet, D. Baykusheva, A. Rupenyan, L. Horný, E. F. Penka, G. Grassi, O. I. Tolstikhin, J. Schneider, F. Jensen, L. B. Madsen, A. D. Bandrauk, F. Remacle and H. J. Wörner, Science 350 (2015) 790.

[7] I. N. Ansari, M. S. Mrudul, M. F. Ciappina, M. Lewenstein and G. Dixit, Phys. Rev. A 98 (2018) 063406.

[8] L. Feng, Phys. Rev. A 92 (2015) 053832.

[9] H. Du, Y. Wen, X. Wang and B. Hu, Opt. Express 21 (2013) 21337.

[10] Z. Zhai, Q. Zhu, J. Chen, Z.-C. Yan, P. Fu and B. Wang, Phys. Rev. A 83 (2011) 043409.

[11] J. B. Watson, A. Sanpera, X. Chen and K. Burnett, Phys. Rev. A 53 (1996) R1962.

[12] A. Sanpera, J. B. Watson, M. Lewenstein and K. Burnett, Phys. Rev. A 54 (1996) 4320.

[13] B. Wang, T. Cheng, X. Li, P. Fu, S. Chen and J. Liu, Phys. Rev. A 72 (2005) 063412.

[14] M. Mohebbi and S. Batebi, Opt. Commun. 296 (2013) 113. 
[15] N.-L. Phan, T.-T. Nguyen, H. Mineo and V.-H. Hoang, JOSA B 37 (2020) 311.

[16] J. Bao, W. Chen, Z. Zhao and J. Yuan, J. Phys. B: At. Mol. Opt. Phys. 44 (2011) 195601.

[17] M. Lein, P. P. Corso, J. P. Marangos and P. L. Knight, Phys. Rev. A 67 (2003) 023819.

[18] S. Baker, J. S. Robinson, M. Lein, C. C. Chirilă, R. Torres, H. C. Bandulet, D. Comtois, J. C. Kieffer, D. M. Villeneuve, J. W. G. Tisch and J. P. Marangos, Phys. Rev. Lett. 101 (2008) 053901.

[19] P. Wei, Y. Yu, X. Guo, X. Ge, P. Liu, Z. Zeng and R. Li, Opt. Express 19 (2011) 147.

[20] M. Lein, Phys. Rev. Lett. 94 (2005) 053004.

[21] H. Shull and G. Hall, Nature 184 (1959) 1559.

[22] J. Zhao and Z. Zhao, Phys. Rev. A 78 (2008) 053414.

[23] T. Sharp, Atomic data 2 (1971) 119.

[24] M. Feit, J. Fleck and A. Steiger, J. Comput. Phys. 47 (1982) 412.

[25] R. Kosloff and H. Tal-Ezer, Chem. Phys. Lett. 127 (1986) 223.

[26] H. Avetissian and G. Mkrtchian, Phys. Rev. A 66 (2002) 033403.

[27] N.-L. T. Phan, P.-H. Tran and V.-H. Hoang, Journal of Science - HCMUE 12 (2016) 5.

[28] G. G. Paulus, W. Becker, W. Nicklich and H. Walther, J. Phys. B: At. Mol. Opt. Phys. 27 (1994) L703.

[29] J. Higuet, H. Ruf, N. Thiré, R. Cireasa, E. Constant, E. Cormier, D. Descamps, E. Mével, S. Petit, B. Pons, Y. Mairesse and B. Fabre, Phys. Rev. A 83 (2011) 053401. 